\title{
Crossover from Isotropic to Directed Percolation
}

\author{
Per Fröjdh and Marcel den Nijs \\ Department of Physics, University of Washington, P.O. Box 351560, Seattle, Washington 98195-1560
}

\begin{abstract}
Directed percolation is one of the generic universality classes for dynamic processes. We study the crossover from isotropic to directed percolation by representing the combined problem as a random cluster model, with a parameter $r$ controlling the spontaneous birth of new forest fires. We obtain the exact crossover exponent $y_{D P}=y_{T}-1$ at $r=1$ using Coulomb gas methods in 2D. Isotropic percolation is stable, as is confirmed by numerical finite-size scaling results. For $D \geq 3$, the stability seems to change. An intuitive argument, however, suggests that directed percolation at $r=0$ is unstable and that the scaling properties of forest fires at intermediate values of $r$ are in the same universality class as isotropic percolation, not only in $2 \mathrm{D}$, but in all dimensions.
\end{abstract}

Directed percolation (DP) has emerged during recent years as one of the most common dynamic universality classes. It applies to a wide array of dynamic processes, ranging from flow through a porous medium in a gravitational field, forest fires and epidemic growth, to surface chemical reactions [1]. In $1+1$ dimensions the DP critical exponents are known accurately from numerical studies in the early eighties [2]. Today the numbers have been refined [3], but analytic insight is still lacking. The ultimate goal is to understand scale invariance in DP at the same level as that of isotropic percolation (IP). This will require some sort of generalization of conformal invariance [4] and the Coulomb gas method [5, 60, which apply to isotropic scaling phenomena in $1+1$ dimensions. The scaling properties of dynamic processes like DP are intrinsically anisotropic. Studies of crossover phenomena, like the one presented here, are a start into this direction.

Consider a square lattice, as shown in Fig. 1. For IP the bond between vertices $i$ and $j$ represents a channel that can be open or closed, $s_{i j}= \pm 1$, with a probability $p$ or $1-p$, respectively. At $p_{c}=\frac{1}{2}$, an infinite cluster of connected channels appears, such that fluid can percolate all the way through the lattice from one end to the other. The correlation length diverges as $\xi \sim\left|p-p_{c}\right|^{-\nu}$ with $1 / \nu=y_{p}=y_{T}=3 / 4$ [6].

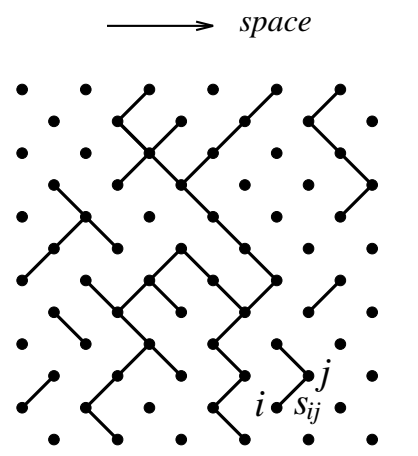

(a)

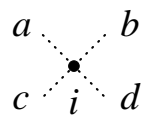

(b)
FIG. 1. Bond percolation on a square lattice with a time-like vertical direction: (a) Open bonds $\left(s_{i j}=1\right)$ are marked with solid lines. (b) Bonds around each vertex $i$ are labeled $s_{a}, s_{b}, s_{c}$, and $s_{d}$.
Directed percolation describes the same type of flow in a porous medium, but in the presence of a gravitational field. The fluid can only flow downwards (positive time direction in Fig. 1). Points at either end are considered connected only if there exists a path between them without the need to back-track in time. The DP threshold is larger, $p_{c}=0.6447$, and the scaling properties at $p_{c}$ are anisotropic. The time-like $(\|)$ and spatial $(\perp)$ correlation lengths diverge with different exponents, $\xi_{\|} \sim\left|p-p_{c}\right|^{-\nu_{\|}}$and $\xi_{\perp} \sim\left|p-p_{c}\right|^{-\nu_{\perp}}$, respectively, with $z=\nu_{\|} / \nu_{\perp}=1.581$ the dynamic critical exponent and $y_{p}=1 / \nu_{\perp}=0.9117$ the scaling dimension of $p-p_{c}$. The values of these exponents are known only numerically [3].

One of the alternate incarnations of DP is as a model for the propagation in time of a forest fire on a $(D-1)$ dimensional lattice. DP treats burnt trees as equivalent to never-burnt trees. This absence of immunization is unrealistic, unless we visualize this as a fire inside a $D$ dimensional forest in a strong wind. The fire propagates only in the direction of the wind, which maps out time. The difference between IP and DP is the possibility to spontaneously ignite new forest fires. Define two operators (see Fig. 1):

$$
\mathcal{C}_{i}=\frac{1}{4}\left(1-s_{a}\right)\left(1-s_{b}\right)\left[1-\frac{1}{4}\left(1-s_{c}\right)\left(1-s_{d}\right)\right]
$$

equals one if a new forest fire is created at vertex $i$ and is zero otherwise.

$$
\mathcal{A}_{i}=\frac{1}{16}\left(1-s_{a}\right)\left(1-s_{b}\right)\left(1-s_{c}\right)\left(1-s_{d}\right)
$$

equals one if vertex $i$ is not part of any forest fire and is zero otherwise. Consider the following partition function

$$
\mathcal{Z}=\sum_{\mathcal{G}} p^{N_{b}}(1-p)^{2 N_{v}-N_{b}} q^{N_{c}} \prod_{i} r^{\mathcal{C}_{i}} \gamma^{\mathcal{A}_{i}}
$$

where $N_{v}$ is the number of vertices of the lattice, $N_{b}$ the number of percolating bonds in graph $\mathcal{G}$, and $N_{c}$ the number of disconnected clusters in $\mathcal{G}$ (including the ones that contain only one vertex). $0 \leq r \leq 1$ controls the spontaneous birth of forest fires, and the factors 


$$
\gamma=\frac{1-r p(2-p)}{(1-p)^{2}}
$$

ensure that the partition function remains stochastic at $q=1$ for all values of $r$. At $r=1$ we recover the conventional random cluster (RC) model [7], which is equivalent to the $q$-state Potts model and describes IP in the $q=1$ limit [8].

At $r=1$ the RC model can be mapped onto the 6 vertex model and in the continuum limit onto a sineGordon type model. The latter can be rephrased as a Coulomb gas between charged plates [6]. These equivalences lead in the early eighties to the derivation of the exact values of the critical exponents of the Potts model [5.6. The same techniques can be applied to determine the stability of IP with respect to $r$. At $r=1$ and $p=\frac{1}{2}$ the crossover operator takes the form

$$
\begin{aligned}
\mathcal{O}_{i} & =\frac{1}{8}\left(s_{a}+s_{b}+s_{c}+s_{d}\right)+\frac{1}{8}\left(s_{c}+s_{d}-s_{a}-s_{b}\right) \\
& -\frac{1}{4}\left[s_{c} s_{d}+2\left(s_{a}+s_{b}\right)\left(s_{c}+s_{d}\right)\right]+O\left(s^{3}\right) .
\end{aligned}
$$

First we need to substract from Eq. (5) operators that are already present in the theory at $p_{c}$ and $r=1$. Those operators are invariant under time-reversal $T\left(s_{a} \leftrightarrow s_{c}\right.$ and $s_{b} \leftrightarrow s_{d}$; see Fig. 1). For example, the spin variables $s_{i j}$ represent the energy density of the Potts model. It is invariant under $T$ and changes sign under the duality transformation $\left(s_{i j} \rightarrow-s_{i j}\right)$. The first term on the right hand side of Eq. (5) is the energy density. Its presence indicates that the critical line approaches $r=1$ with a finite angle. The second term has the form of the gradient of the energy operator with respect to time, and breaks time reversal. This is the true crossover operator. It is essential not to miss any topological operators that might be hiding in $\mathcal{O}_{i}$. This is where the mapping to the Coulomb gas becomes important. We have determined the Coulomb gas representation of the crossover-operator pair-correlation function. The details are not presented here, since we find no hidden topological operators. The most dominant component in $\mathcal{O}_{i}$ that breaks time reversal is simply the gradient of the energy operator with respect to time,

$$
\mathcal{O}_{i} \sim \frac{\partial \epsilon}{\partial t}
$$

Hence, the crossover exponent is equal to $y_{D P}=y_{T}-1$. This is an exact result. The crossover exponent is irrelevant, $y_{D P}=-1 / 4$ is negative since $y_{T}=3 / 4$. This indicates that the scaling properties of a single forest fire, DP at $r=0$, are different from those of forests in which new fires can spontaneously ignite, with probability proportional to $r$. Those must have the same scaling properties as IP. The following numerical results confirm this.

We have performed a finite-size scaling (FFS) analysis of Eq. (3) at $q=1$. The transfer matrix acts on a state vector which in its most efficient representation contains only slightly less than $5^{N}$ elements for system size $N$. Non-local connectivity information is required at each moment in time. It does not suffice to know which trees are on fire and which are not. We need to know which burning trees at time $t$ are part of the same cluster via a path in the past. Our coding for this is inspired by an earlier FSS analysis at $r=1$ by Blöte and Nightingale [9]. The full connectivity can be reconstructed by attributing to each vertex 5 possible states: $(0, \Leftarrow, \Rightarrow, \Leftrightarrow, \star)$. Vertex $i$ is not part of any forest fire (state 0 ) if both trees just above it are not burning, $s_{a}=s_{b}=-1$ (see Fig. 1), but is part of the fire if either or both are burning. In state $\Leftarrow$ at least one vertex to the left of $i$ is part of the same cluster, but none to its right. In state $\Rightarrow$ at least one vertex to the right is part of the same cluster, but none to its left. In state $\Leftrightarrow$ at least two vertices, one on each side, is part of the same cluster. In state $\star$ no other vertices at this moment in time are part of the same cluster.

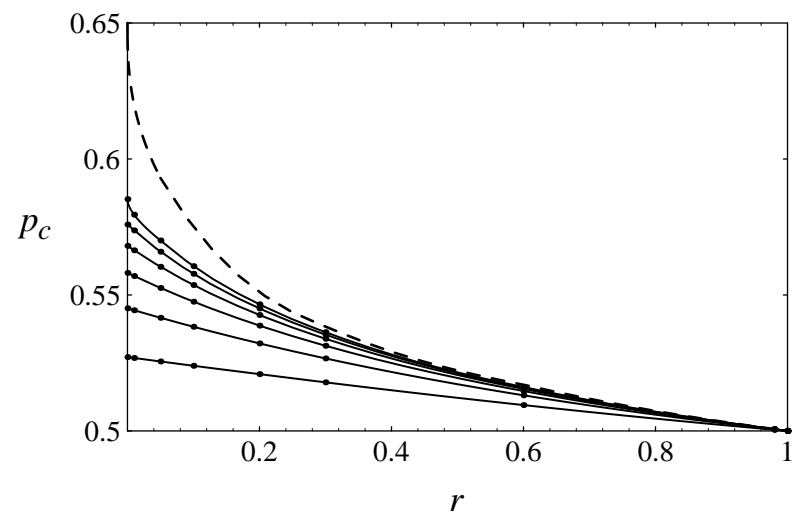

FIG. 2. Finite-size estimates for the percolation threshold $p_{c}(r)$ from the locations of the minima in the energy mass gap. Results for system sizes $N=3, \ldots, 8$ (solid lines from bottom to top) are extrapolated to infinite $N$ (dashed line).

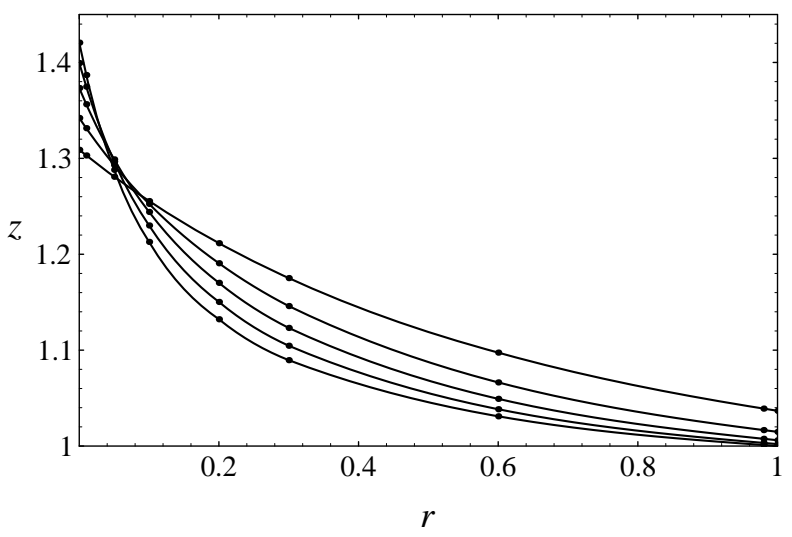

FIG. 3. Finite-size estimates $z(N, N+1)$ for the dynamic exponent $z$ using the relation $m \simeq A / N^{z}$. For $r>0$, $z(3,4), \ldots, z(7,8)$ (from top to bottom) converge to $z=1$. 
Fig. 2 shows FSS estimates for the location of the percolation threshold $p_{c}(r)$ for system sizes $3 \leq N \leq 8$. The leading and next-leading eigenvalues of the transfer matrix give us the energy mass gap, $m_{e}=\xi_{\|}^{-1}$, the inverse of the time-like correlation length of energy-energy correlations. This mass gap is finite at either side of $p_{c}$. The minima in $m_{e}$ with respect to $p$ at each $r$ shown in Fig. 2 converge to $p_{c}(r)$.

Fig. 3 shows FSS estimates for the dynamic exponent $z$. At $p_{c}$ the energy mass gap scales with system size as $m_{e} \simeq A / N^{z}$. The values of the mass gap at the minima shown in Fig. 2, yield for each set of system sizes $(\mathrm{N}, \mathrm{N}+1)$ a FSS estimate for $z$. These estimates converge to the IP value $z=1$ for all $r$, except very close to $r=0$. Crossover scaling toward the DP value $z=1.581$ does not set in until $r$ is rather small. FSS estimates for $y_{p}=1 / \nu_{\perp}$ (not shown here) can be obtained from the derivatives of $m_{e}$ with respect to $p$ at the minima. These behave similarly to the estimate for $z$ in Fig. 3 and converge towards the IP value $y_{p}=y_{T}=3 / 4$ for all $r>0$. Crossover scaling towards the DP value does not play a role until rather small values of $r$. These numerical results clearly confirm that for all $r>0$ the scaling properties are identical to those of IP.

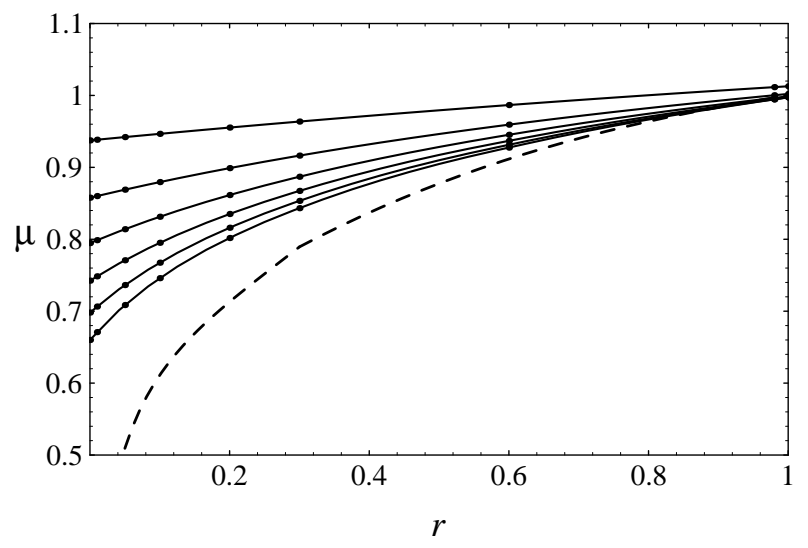

FIG. 4. Finite-size estimates for the anisotropy parameter $\mu=\xi_{\perp} / \xi_{\|}$at $p_{c}$ using the relation $m \simeq 2 \pi \mu\left(2-y_{T}\right) / N$ for system sizes $N=3, \ldots, 8$ (solid lines from top to bottom). The dashed line corresponds to the extrapolation $N \rightarrow \infty$.

Fig. 4 shows the FSS estimates for the aspect ratio $\mu=\xi_{\perp} / \xi_{\|}$at $p_{c}(r)$. Both correlation lengths diverge with exponent $\nu=4 / 3$, but their amplitudes differ by a factor $\mu$. We obtain $\mu$ from the energy mass gap. It scales according to conformal field theory as $m_{e} \simeq A / N$ with a universal amplitude $A=2 \pi \mu\left(2-y_{T}\right)$. In Fig. 4 we plot $N m_{e} / 2 \pi\left(2-y_{T}\right)$ (with $\left.y_{T}=3 / 4\right)$ at the minima shown in Fig. 2. For DP this ratio represents the so-called opening angle [2] and goes to zero at $p_{c}$. The convergence to a non-zero value for $r>0$ gives further confirmation that the scaling is isotropic, $z=1$.

It is a safe guess that Eq. (6) and $y_{D P}=y_{T}-1$ hold also in dimensions larger than $D=2$. Eq. (5) is easily generalized to higher dimensions. The only aspect missing for general $D$ is the Coulomb-gas rigorous proof that the scaling dimension of the gradient of the energy maintains its naive value $y_{T}-1$. The relation $y_{D P}=y_{T}-1$ suggests that IP changes stability with dimensionality. The critical exponents for IP in 3D are known numerically [10]; $y_{T}=1.14$ yields $y_{D P}=0.14$. So for $D \geq 3$ the crossover operator appears to be relevant. Mean-field theory, valid in $D \geq 6$, predicts that the crossover exponent is relevant as well. Power counting yields the value $y_{D P}=1$.

Our identification of the crossover scaling field with the energy gradient agrees qualitatively with a recent fieldtheoretical analysis by Frey et al. 111] (see also Ref. [12]). They describe IP by a $\phi^{3}$-type theory similar to the conventional one for the $q$-state Potts model, and set up an interpolation scheme between this and Reggeon field theory [13], which belongs to the DP universality class. Frey et al. perform a one-loop expansion about the upper critical dimension $D_{u}=6$ for IP and observe indeed a flow from isotropic to directed percolation in their field theory. Our approach has the advantage of being based on a well-defined microscopic model, and being exact in 2D.

The apparent turn-about in the stability of IP for $2<D<3$ is the most intriguing implication of Eq. (6). Initially we thought that IP should be unstable towards DP in all dimensions. $r<1$ introduces a preferred direction in the model (the time-like direction). This breaks a fundamental symmetry of the problem and therefore it seems logical that the scaling properties change. This is too simplistic, however. The crossover operator is the gradient of the energy density. $y_{T}$ is the fractal dimension of so-called "red vertices" [14, i.e., of vertices where the cluster is singly connected (like the $\star$ states in our transfer matrix). Such vertices are rare, to the extent that in $D=2$ their fractal dimension becomes smaller than one, $y_{T}=3 / 4$. This causes $y_{D P}$ to become negative.

The following argument puts the shoe on the other foot. It supports the opposite point of view, namely that DP at intermediate values of $r$ belongs to the same universality class as IP (at $r=1)$ in all dimensions. Consider DP at $r=0$ just below its percolation threshold. All forest fires that are initially burning die out. They are finite in size with a characteristic aspect ratio $\mu_{0}=\ell_{\perp} / \ell_{\|}$. At small non-zero $r$, new forest fires are starting at all times $t$. They have the same typical size and aspect ratio as those at $r=0$. The probability that a specific vertex belongs to a forest fire is of order $\tilde{p} \simeq r \ell_{\perp}^{D-1} \ell_{\|}$. These forest fires are placed at random, and increasing $r$ is equivalent to increasing $\tilde{p}$ in a conventional percolation problem, one in which objects of a specific size and shape are placed at random in space. Let $\tilde{p}_{c}$ be the percolation threshold for that percolation problem. The scaling properties at $\tilde{p}_{c}$ are identical to those at $r=1$. 
The above argument suggests that DP is always unstable with respect to the creation of spontaneous forest fires. It is probably too simplistic to treat $\tilde{p}_{c}$ as a constant, but doing so leads to the following estimate for the crossover exponent $y_{I P}$ at $r=0$. The IP critical line approaches the DP critical point at $r=0$ as $\tilde{p}_{c} \simeq r \ell_{\perp}^{D-1} \ell_{\|} \sim r\left(p-p_{c}\right)^{-(z+D-1) \nu_{\perp}}$ with $p_{c}$ the DP percolation threshold at $r=0$. According to conventional scaling theory the critical line must approach the DP critical point along a renormalization flow line. This yields $y_{I P}=z+D-1$ since $y_{p}=1 / \nu_{\perp}$. This value can only be an approximation, since it violates the fundamental rule that critical dimensions, like $y_{I P}$, cannot be larger than the embedded dimension. It shows however that DP is strongly unstable with respect to $r$ for all $D$.

This leaves us with a puzzle. Do the scaling properties at intermediate values of $r$ change between $D=2$ and $D=3$, as suggested by the stability analysis at $r=1$, Eq. (6), or not, as suggested by the above argument close to $r=0$ ? Future numerical studies in $D=3$ will decide this issue. For the time being, we put our bets on the above argument. It is hard to see how it can be circumvented. Moreover, the gradient nature of the crossover operator at $r=1$ is suspicious. Gradient operators can be integrated up into boundary operators and then often vanish from the theory altogether. This would leave the crossover at $r=1$ irrelevant in all $D$.

This research is supported by NSF grant DMR9205125 .
[1] For a discussion on the applicability of directed percolation, see P. Grassberger, J. Stat. Phys. 79, 13 (1995).

[2] W. Kinzel, in Percolation Structures and Processes, edited by G. Deutscher, R. Zallen, and J. Adler, Annals of the Israel Physical Society, Vol. 5 (Adam Hilger, Bristol, 1983).

[3] I. Jensen, J. Phys. A: Math. Gen. (in press, 1996).

[4] J. L. Cardy, in Phase Transitions and Critical Phenomena, vol. 11, edited by C. Domb and J. L. Lebowitz (Academic Press, London, 1987).

[5] B. Nienhuis, in Phase Transitions and Critical Phenomena, vol. 11, edited by C. Domb and J. L. Lebowitz (Academic Press, London, 1987).

[6] M. den Nijs, J. Phys. A: Math. Gen. 12, 1857 (1979) and Phys. Rev. B 27, 1674 (1983).

[7] C. M. Fortuin and P. W. Kasteleyn, Physica 57, 536 (1972).

[8] F. Y. Wu, Rev. Mod. Phys. 54, 235 (1982).

[9] H. W. J. Blöte and M. P. Nightingale, Physica 112A, 405 (1982).

[10] N. S. Branco and K. D. Machado, Phys. Rev. B 47, 493 (1993).

[11] E. Frey, U. C. Täuber, and F. Schwabl, Phys. Rev. E 49, 5058 (1994).

[12] A. A. Luzhkov, JETP Lett. 62, 326 (1995).

[13] J. L. Cardy and R. L. Sugar, J. Phys. A: Math. Gen. 13, L423 (1980).

[14] A. Bunde and S. Havlin, in Fractals and Disordered Systems, edited by A. Bunde and S. Havlin (Springer-Verlag, Heidelberg, 1991). 